\title{
Role of the transcriptional regulator SP140 in resistance to bacterial infections via repression of type $I$ interferons
}

Daisy X. Ji ${ }^{1}$, Kristen C. Witt ${ }^{1}$, Dmitri I. Kotov ${ }^{1,2}$, Shally R. Margolis ${ }^{1}$, Alexander Louie ${ }^{1}$, Katherine J. Chen ${ }^{1,2}$, Harmandeep S. Dhaliwal ${ }^{3}$, Angus Y. Lee ${ }^{3}$, Dario S. Zamboni ${ }^{4}$, Igor Kramnik $^{5}$, Daniel A. Portnoy ${ }^{1,6,7}$, K. Heran Darwin ${ }^{8}$, Russell E. Vance ${ }^{1,2^{*}}$

${ }^{1}$ Division of Immunology and Pathogenesis, Department of Molecular and Cell Biology, University of California, Berkeley, CA 94720 USA

${ }^{2}$ Howard Hughes Medical Institute, University of California, Berkeley, CA 94720 USA

${ }^{3}$ Cancer Research Laboratory, University of California, Berkeley, CA 94720 USA

${ }^{4}$ Department of Cell Biology, Ribeirão Preto Medical School, University of São Paulo, Ribeirão Preto, São Paulo, Brazil.

${ }^{5}$ The National Emerging Infectious Diseases Laboratory, Department of Medicine (Pulmonary Center), and Department of Microbiology, Boston University School of Medicine, Boston, MA, USA.

${ }^{6}$ Division of Biochemistry, Biophysics and Structural Biology, Department of Molecular and Cell Biology, University of California, Berkeley, CA 94720 USA

${ }^{7}$ Department of Plant and Microbial Biology, University of California, Berkeley, CA 94720 USA

${ }^{8}$ Department of Microbiology, New York University School of Medicine, New York, NY, USA

* e-mail: rvance@berkeley.edu 


\begin{abstract}
Type I interferons (IFNs) are essential for anti-viral immunity, but often impair protective immune responses during bacterial infections. How type I IFNs are strongly induced during viral infections, and yet are appropriately restrained during bacterial infections, remains poorly understood. The Super susceptibility to tuberculosis 1 (Sst1) locus in mice confers resistance to many bacterial infections. Here we provide evidence that $S p 140$ is a gene encoded within the Sst1 locus that functions to repress the expression of type I IFNs during bacterial infections. We generated $S p 140^{--}$mice and find they are susceptible to infection by diverse bacteria, including Listeria monocytogenes, Legionella pneumophila, and Mycobacterium tuberculosis.

Susceptibility of $S p 140^{-1-}$ mice to bacterial infection was rescued by crosses to mice lacking the type I IFN receptor (Ifnar ${ }^{--}$). Our results implicate $S p 140$ as an important repressor of type I IFNs that is essential for resistance to bacterial infections.
\end{abstract}

\title{
Introduction
}

Type I interferons (IFNs) comprise a group of cytokines, including interferon- $\beta$ and multiple interferon- $\alpha$ isoforms, that are essential for immune defense against most viruses (Stetson and Medzhitov, 2006). Type I IFNs signal through a single cell surface receptor, the interferon alpha and beta receptor (IFNAR), to induce an 'anti-viral state' that is characterized by the transcriptional induction of hundreds of interferon stimulated genes (ISGs) (Schneider et al., 2014). Many ISGs encode proteins with direct anti-viral activities. Type I IFNs also promote anti-viral responses by cytotoxic $\mathrm{T}$ cells and Natural Killer cells. Accordingly, Ifnar ${ }^{-1-}$ mice are highly susceptible to most viral infections.

Many ISGs are also induced by IFN- $\gamma$ (also called type II IFN). However, type I and type II IFNs appear to be specialized for the control of different classes of pathogens (Crisler and Lenz, 2018). In contrast to the predominantly anti-viral activity of type I IFNs, the subset of ISGs induced preferentially by IFN- $\gamma$ appears to be especially important for the control of intracellular bacteria and parasites. By contrast, type I IFNs play complex roles during bacterial infections (Boxx and Cheng, 2016; Donovan et al., 2017; McNab et al., 2015; Moreira-Teixeira et al., 2018). Some ISGs induced by type I IFN, most notably certain guanylate binding proteins (GBPs), have anti-bacterial activities (Pilla-Moffett et al., 2016). At the same time, several proteins induced by type I IFNs, including interleukin-10 (IL-10) and IL-1 receptor antagonist (IL-1RA), impair anti-bacterial immunity (Boxx and Cheng, 2016; Ji et al., 2019; Mayer-Barber et al., 2014). As a result, the net effect of type I IFN is often to increase susceptibility to bacterial infections. For example, Ifnar ${ }^{-/-}$mice exhibit enhanced resistance to Listeria monocytogenes (Auerbuch et al., 2004; Carrero et al., 2004; O'Connell et al., 2004) and Mycobacterium tuberculosis (Donovan et al., 2017; Dorhoi et al., 2014; Ji et al., 2019; Mayer-Barber et al., 2014; Moreira-Teixeira et al., 2018; Stanley et al., 2007). Multiple mechanisms appear to explain resistance of Ifnar ${ }^{-1-}$ mice to L. monocytogenes, including a negative effect of type I IFNs on protective IFN- $\gamma$ signaling (Rayamajhi et al., 2010). Likewise, diverse mechanisms underlie the negative effects of type I IFNs during $M$. tuberculosis infection, including alterations of eicosanoid production (Mayer-Barber et al., 2014) and the induction of IL-1Ra (Ji et al., 2019), both of which impair protective IL-1 responses.

As an experimental model for dissecting the mechanisms by which inappropriate type I IFN responses are restrained during bacterial infections, we have compared mice harboring different haplotypes of the Super susceptibility to tuberculosis 1 (Sst1) locus (Pan et al., 2005; 
Pichugin et al., 2009). The Sst1 locus encompasses a region of about $10 \mathrm{M}$ base pairs of mouse chromosome 1 that contains approximately 50 genes. Mice harboring the susceptible $(S)$ haplotype of Sst1, derived from the $\mathrm{C} 3 \mathrm{H} / \mathrm{HeBFeJ}$ mouse strain, succumb relatively rapidly to $M$. tuberculosis infection, as compared to isogenic mice harboring the resistant (R) Sst 1 haplotype (derived from C57BL/6 mice). Likewise, $S s t 1^{S}$ mice also exhibit enhanced susceptibility to Listeria monocytogenes (Boyartchuk et al., 2004; Pan et al., 2005) and Chlamydia pneumoniae (He et al., 2013). The susceptibility of $S s t 1^{S}$ mice to both M. tuberculosis and C. pneumoniae was reversed by crossing to Ifnar ${ }^{-\gamma}$ mice (He et al., 2013; Ji et al., 2019), thereby demonstrating the causative role of type I IFNs in driving the susceptibility phenotype. Although multiple type I IFN-induced genes are likely responsible for the detrimental effects of type I IFNs during bacterial infections, heterozygous deficiency of a single type I IFN-induced gene, Illrn (encoding IL-1 receptor antagonist), was sufficient to almost entirely reverse the susceptibility of Sst $1^{S}$ mice to M. tuberculosis (Ji et al., 2019).

The $S s t 1^{R}$ haplotype is dominant over the $S_{s t} 1^{S}$ haplotype, suggesting that $S_{s} t 1^{R}$ likely encodes a protective factor that is absent from $S s t 1^{S}$ mice (Pan et al., 2005; Pichugin et al., 2009). By comparing gene expression in $S s t 1^{R}$ versus $S s t 1^{S}$ mice, $S p 110$ (also known as Iprl) was discovered as an $S s t 1$-encoded gene that is transcribed selectively in $S_{s t} 1^{R}$ mice (Pan et al., 2005). Transgenic expression of $S p 110$ in $S s t 1^{S}$ mice partially restored resistance to $M$. tuberculosis and L. monocytogenes (Pan et al., 2005). However, the causative role of Sp110 in conferring resistance to bacterial infections was not confirmed by targeted deletion of $S p 110$ from B6 mice.

In humans and mice, SP110 is a part of the Speckled Protein (SP) family of nuclear proteins, consisting of SP100, SP110 and SP140 (and SP140L in humans and primates only) (Perniola and Musco, 2014). The SP family members also exhibit a high degree of similarity to AIRE, a transcriptional regulator that promotes tolerance to self-antigens by inducing their expression in thymic epithelial cells (Anderson and Su, 2016; Perniola and Musco, 2014). All members of the SP-AIRE family in both mice and humans have an N-terminal SP100 domain that appears to function as a homotypic protein-protein interaction domain. The SP100 domain is closely related to the Caspase Activation and Recruitment Domain (CARD), though SP family members are not believed to activate caspases. SP-AIRE proteins also contain a DNA-binding SAND domain (Bottomley et al., 2001). Certain SP isoforms, including all human full-length SP family members and mouse SP140, also include a plant homeobox domain (PHD) and a bromodomain (BRD) (Perniola and Musco, 2014). The genes encoding SP family proteins are linked in a small cluster in both mouse and human genomes and are inducible by IFN- $\gamma$ in a variety of cell lines. The mouse $S p 100 / 110 / 140$ gene cluster is adjacent to a highly repetitive 'homogenously staining region' of chromosome 1 that remains poorly assembled in the most recent genome assembly due to the presence of as many as 40 near-identical repeats of $S p 110$ like sequences (Pan et al., 2005; Weichenhan et al., 2001). These repeated Sp110-like sequences appear to be pseudogenes and are not believed to be translated, but their presence has nevertheless complicated genetic targeting and analysis of the SP gene family.

Null mutations of human SP110 are associated with VODI (hepatic veno-occlusive disease with immunodeficiency syndrome, OMIM 235550), but not mycobacterial diseases (Roscioli et al., 2006). Some studies have found polymorphisms in SP110 to be associated with susceptibility to TB, though not consistently so across different ethnic groups (Chang et al., 2018; Fox et al., 2014; Lei et al., 2012; Png et al., 2012; Thye et al., 2006; Tosh et al., 2006; Zhang et al., 2017). To address the in vivo function of Sp110, we used CRISPR/Cas9-based methods (Wang et al., 2013) to generate $S p 110^{-1-}$ mice on the B6 background. Surprisingly, we found that $S p 110^{-1-}$ mice do not phenocopy the susceptibility of $S s t 1^{S}$ mice to M. tuberculosis 
infection in vivo. Instead, we found that B6.Sst $1^{S}$ mice also lack expression of $S p 140$. To test whether loss of $S p 140$ might account for the susceptibility of $S s t 1^{S}$ mice to bacterial infections, we generated $S p 140^{-1-}$ mice. We found these mice were as susceptible as B6.Sst $1^{S}$ mice to $M$. tuberculosis, L. monocytogenes and another intracellular pathogen, Legionella pneumophila. Similar to B6.Sst $1^{S}$ mice, $S p 140^{---}$mice exhibited an exacerbated type I IFN response after bacterial infection, and the susceptibility of $S p 140^{-1-}$ mice was rescued by crosses to Ifnar ${ }^{--}$ mice. Our results suggest that loss of Sp140 explains the susceptibility to bacterial infections associated with the $S s t 1^{S}$ haplotype. These data further suggest that SP140 is a novel negative regulator of type I IFN responses during intracellular bacterial infections.

\section{Results}

Sp110 ${ }^{--}$mice are not susceptible to M. tuberculosis. Loss of $S p 110$ expression was proposed to account for the susceptibility of mice carrying the $S s t l^{S}$ haplotype to bacterial infections (Pan et al., 2005). We first used three different antibodies to confirm that bone marrow-derived macrophages (BMMs) from B6.Sst $1^{S}$ mice lack expression of $S p 110$ protein (Figure 1A). To determine whether loss of $S p 110$ confers susceptibility to bacterial infections, we used CRISPR/Cas9 to target exon 4 of $S p 110$ to generate $S p 110^{-1-}$ mice on the C57BL/6 (B6) background (Figure S1). We generated three independent $S p 110^{-1-}$ lines, denoted as lines 61, 65 and 71 (Figure 1A, S1). All three lines lacked expression of SP110 protein (Figure 1A). Sp $110^{-}$ mice are viable and are born at normal Mendelian ratios and litter sizes. Surprisingly, when aerosol infected with a low-dose of M. tuberculosis, Sp 110 ${ }^{-/-}$mice did not phenocopy the susceptibility observed in B6.Sst $1^{S}$ mice (Figure 1B-D). At day 25 post-infection, $S p 110^{-1-}$ lungs resembled those of wild-type B6 mice (Figure 1B) and had lower bacterial burdens than the B6.Sst ${ }^{S}$ mice, similar to both the B6 and $S p 110^{+-}$littermates (Figure 1C). Likewise, the survival of infected $S p 110^{--}$mice was indistinguishable from B6 mice, and mice of both genotypes survived considerably longer than the B6.Sst $1^{S}$ mice (Figure 1D). Thus, despite the clear absence of $S p 110$ from $S s t 1^{S}$ mice, our results indicate that the loss of $S p 110$ is not sufficient to replicate the susceptibility to M. tuberculosis associated with the $S s t 1^{S}$ locus.

$S p 140^{-/-}$mice are susceptible to bacterial infections. Given that $S p 110$ deficiency did not phenocopy the susceptibility of $S s t I^{S}$ mice, we asked whether any other genes found within the Sst1 locus differ in expression between B6 and B6.Sst $1^{S}$ BMMs. Interestingly, a homolog of Sp110 called Sp140 was also reduced in expression in B6.Sst $1^{S}$ cells compared to B6 cells (Figure 2A). Immunoblot confirmed that neither untreated nor IFN- $\gamma$ treated BMMs from B6.Sst $1^{S}$ mice produce SP140 protein (Figure 2B). We used CRISPR/Cas9 to generate two independent lines of $\mathrm{Sp} 140^{-1-}$ mice on a pure B6 background (Figure S2A-D). These mice lack expression of SP140 protein (Figure 2B) but retain the production of SP110 protein (Figure $\mathrm{S} 2 \mathrm{D})$. Like $S p 110^{--}$mice, $S p 140^{---}$mice are viable, fertile and born at the expected Mendelian ratios. However, when infected with $M$. tuberculosis, $S p 140^{-/-}$mice exhibited high bacterial burdens in their lungs at day 28 post-infection, similar to B6.Sst $1^{S}$ mice but significantly greater than B6, Sp110 $0^{-/-}$or $S p 140^{+/-}$littermate mice (Figure 2C, S2E). The increased susceptibility of $S p 140^{-/-}$mice was accompanied by significant weight loss, again phenocopying the B6.Sst ${ }^{S}$ mice (Figure 2D). Both of the independent lines of $S p 140^{--}$were similarly susceptible to $M$. tuberculosis (Figure S2E). Previous reports have shown that in addition to M. tuberculosis, the Sst1 locus also controls responses against other intracellular bacterial pathogens such as $L$. monocytogenes and C. pneumoniae (Boyartchuk et al., 2004; He et al., 2013; Pichugin et al., 2009). Therefore we infected $S p 140^{--}$and B6.Sst $1^{S}$ mice with L. monocytogenes. As previously 
bioRxiv preprint doi: https://doi.org/10.1101/2020.01.07.897553; this version posted January 8, 2020. The copyright holder for this preprint (which was not certified by peer review) is the author/funder, who has granted bioRxiv a license to display the preprint in perpetuity. It is made
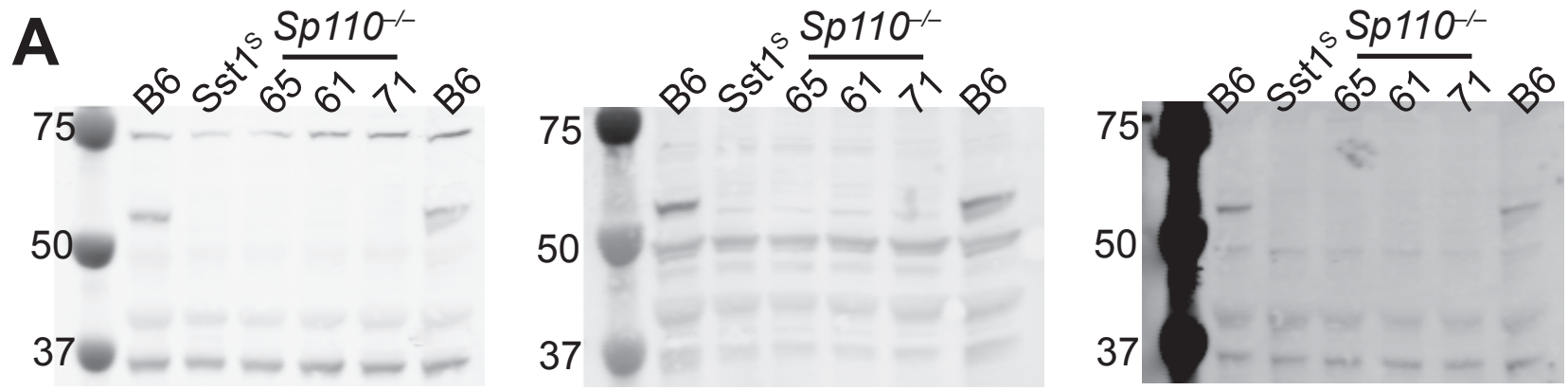

anti-SP110 rabbit polyclonal 1 anti-SP110 rabbit polyclonal 2

anti-SP110 monoclonal

B
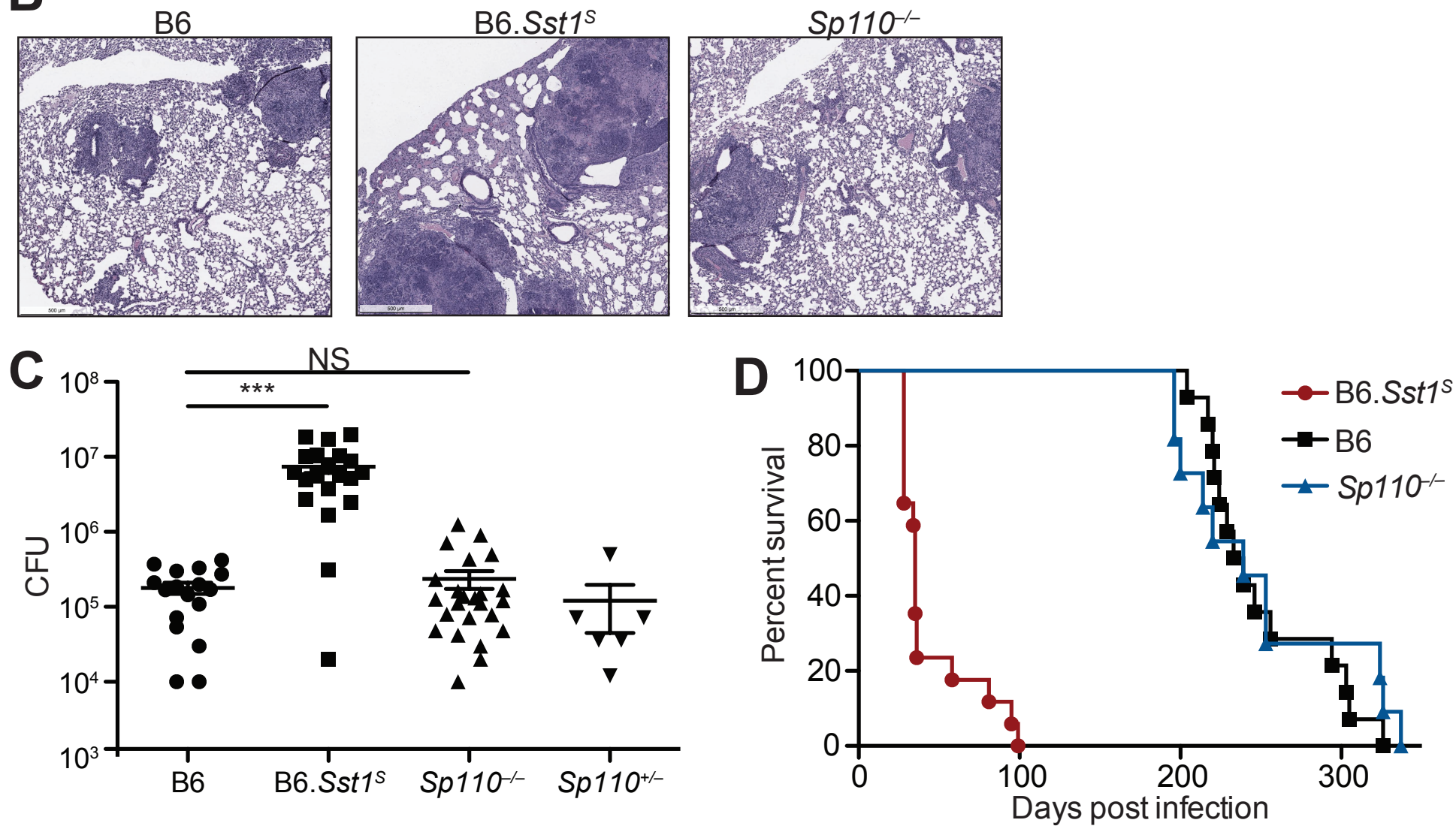

Figure 1. Sp110 $10^{--}$mice are not susceptible to M. tuberculosis infections. (A), BMMs were treated with $10 \mathrm{U} / \mathrm{ml}$ of IFNy for 24 hours and cells were lysed with RIPA buffer. $5 \mu \mathrm{g}$ of total protein was loaded on each lane, and immunoblot was performed with respective antibodies as shown. Molecular weight standards are shown on the left of each blot in $\mathrm{kDa}$. Individual membranes were imaged separately. Three independent lines of Sp100-- mice were analyzed (denoted lines 61, 65, and 71). (B-D), Lung of mice infected with M. tuberculosis were stained with hematoxylin and eosin (H\&E) for histology (B), measured for CFU at 25 days post-infection (Mann-Whitney test) (C) or, monitored for survival (D). All except B6 mice were bred in-house, and combined results from the three independent Sp110 $0^{-/}$lines are shown. Representative of 2 experiments (B, D); combined results of 3 infections (C). ${ }^{*}, p \leq 0.05 ;{ }^{* *}, p \leq$ $0.01 ;{ }^{* * *}, p \leq 0.005$. 
A

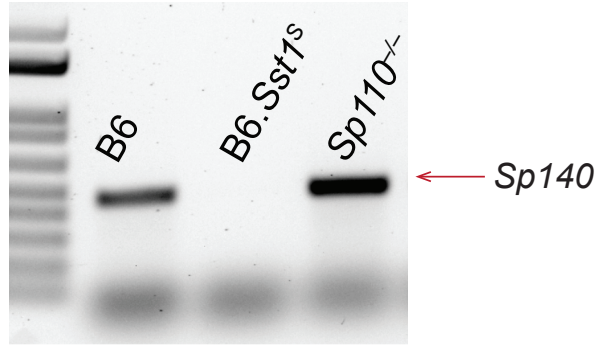

C

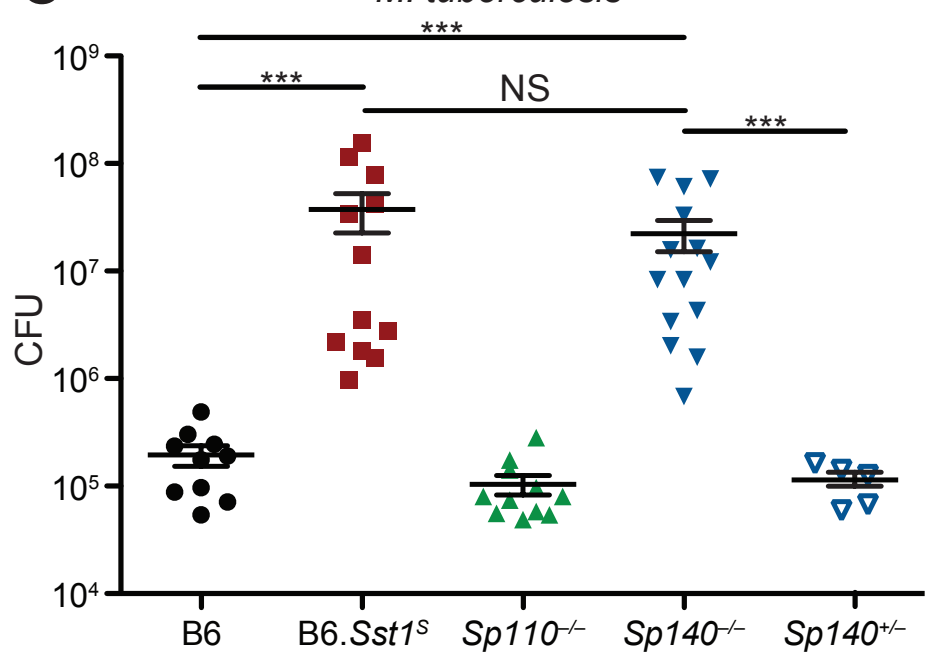

Lung, 28 days post-infection

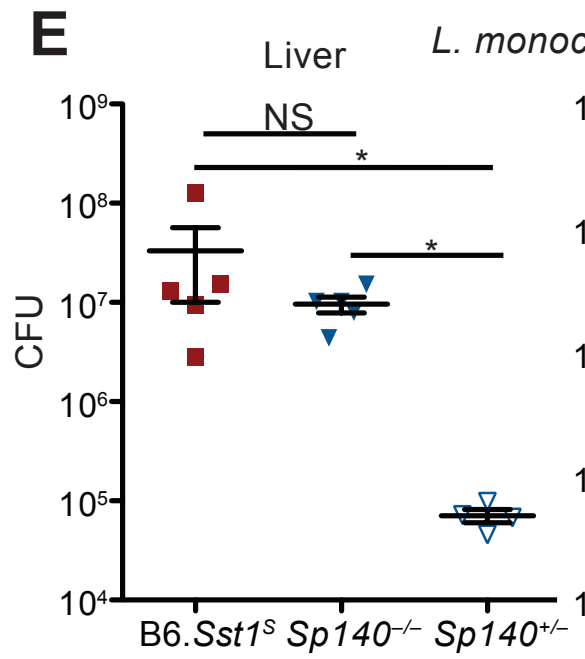

48 hours post-infection
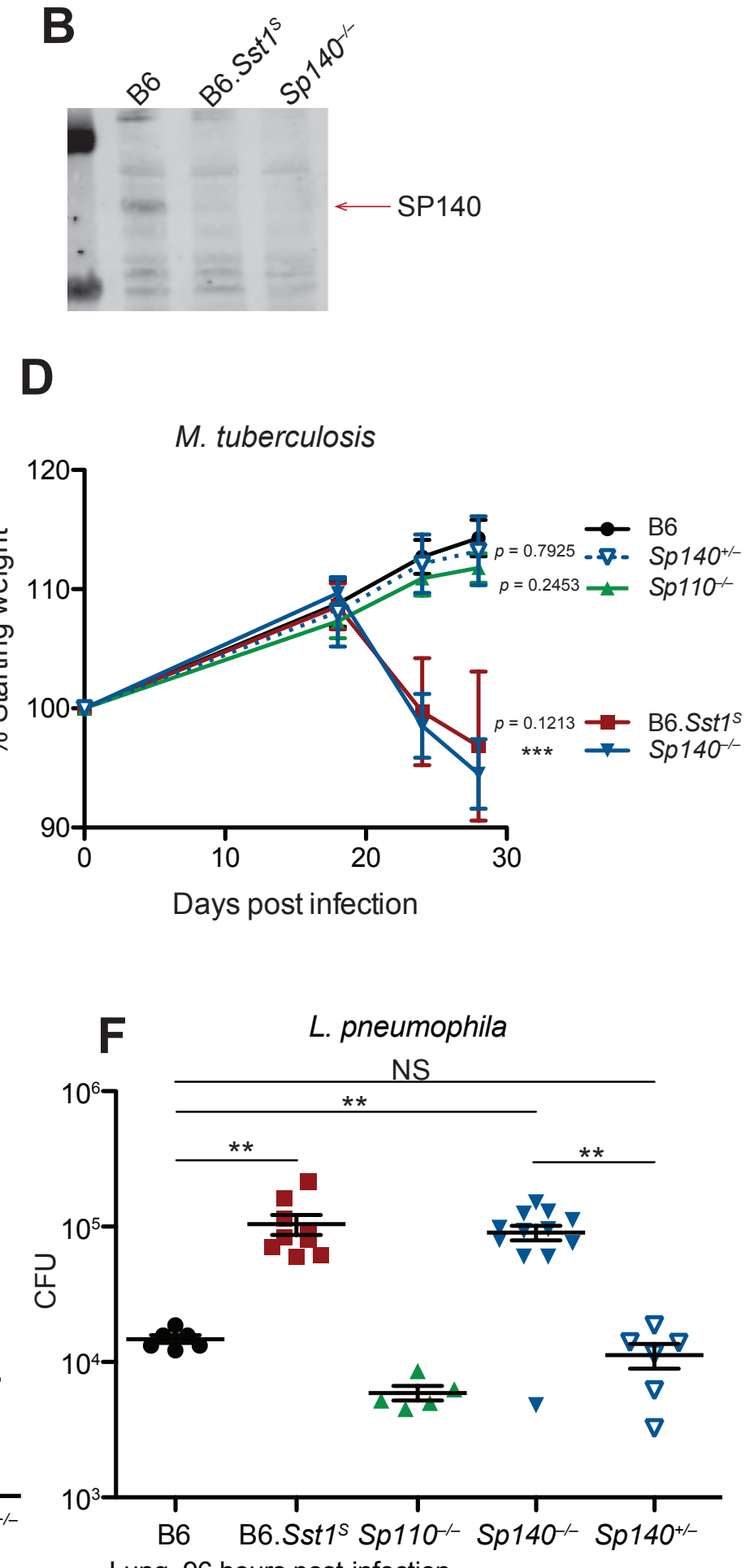

Lung, 96 hours post-infection

Figure 2. Sp140-- mice are susceptible to bacterial pathogens. (A) RT-PCR of cDNA from BMMs of the indicated genotypes. Red arrow indicates band corresponding to a portion of Sp140, verified by sequencing. (B) BMMs of wildtype and Sp140-- mice were treated with $10 \mathrm{U} / \mathrm{ml}$ of recombinant mouse IFNy for 24 hours. Cells were lysed for protein, and equal amounts were loaded for immunoblot with anti-SP140 antibody. (C-D) Mice were infected with M. tuberculosis and measured for lung CFU at 28 days post-infection (C) or body weight over time (D). Statistics in (D) shows comparison to B6 at day 28. (E) Mice were infected with $L$. monocytogenes and tissues were measured for CFU at 48 hours post-infection. (F) Mice were infected with $L$. pneumophila and lungs were measured for CFU at 96 hours post-infection. All mice were bred in-house, Sp140-- and Sp140+- were littermates (C-F). C, D, F are combined results of 2 independent infections. A-D shows representative analysis of one $S p 140^{-/}$line (line 1), whereas E-F includes a mixture of both line 1 and 2. Results of infection of both lines with $M$. tuberculosis is shown in Figure S2E. (C-F) Mann-Whitney test. * $p \leq 0.05 ;{ }^{* *}, p \leq 0.01 ;{ }^{* * *}, p \leq 0.005$. 
reported (Boyartchuk et al., 2004; Pichugin et al., 2009), B6.Sst $1^{S}$ mice were more susceptible to L. monocytogenes, and Sp140 ${ }^{--}$mice had similarly increased bacterial burden in their liver and spleen as compared to the $S p 140^{+/-}$control mice (Figure 2E). We also found that both B6.Sst $1^{S}$ and $S p 140^{--}$mice were more susceptible to the intracellular Gram-negative bacterium Legionella pneumophila, as compared to the B6 and $S p 110^{-/-}$mice (Figure 2F). Collectively our results demonstrate that B6.Sst $1^{S}$ mice lack expression of Sp140, and that the loss of Sp140 on the B6 background appears to phenocopy the broad susceptibility of B6.Sst $1^{S}$ mice to Grampositive, Gram-negative and mycobacterial infections.

Enhanced type I IFN responses in $S p 140^{-/-}$and B6.Sst ${ }^{S}$ mice. We and others previously reported that TNF $\alpha$ induces higher levels of type I IFN and ISGs in $S s t 1^{S}$ BMMs as compared to B6 BMMs (Bhattacharya et al., 2018; Ji et al., 2019). We also observed higher levels of Ifnb transcripts in the lungs of B6.Sst $1^{S}$ mice infected with $M$. tuberculosis, as compared to infected B6 mice (Ji et al., 2019). Similar to B6.Sst $1^{S}$ BMMs, Sp 140 ${ }^{-1-}$ BMMs also exhibited elevated expression of Ifnb and interferon-stimulated genes (ISGs) when stimulated with TNF $\alpha$ (Figure 3A). When infected with M. tuberculosis, the lungs of $S p 140^{-1-}$ and B6.Sst $1^{S}$ mice also exhibited higher levels of Ifnb transcript as compared to B6, Sp110 ${ }^{-/-}$and $S p 140^{+-}$littermate mice (Figure 3B). Likewise, during L. pneumophila infection, $S p 140^{-1-}$ mice expressed more Ifnb in their lungs, as compared to B6 mice. Importantly, elevated Ifnb was evident at 48 hours post-infection when there is no difference in bacterial burdens between the genotypes, and at 96 hours postinfection, when $S p 140^{--}$mice have greater bacterial burdens (Figure 3C).

Infected $S p 140^{-/-}$and B6.Sst ${ }^{S}$ lungs show similar gene expression patterns. We used RNA sequencing to analyze the global gene expression patterns in $M$. tuberculosis-infected lungs of B6, Sp $110^{-/-}, S p 140^{-/-}$and B6.Sst $I^{S}$ mice at day 28 post-infection (Figure 4). Principal component analysis revealed that while there is spread between individual samples, the expression pattern of $S p 140^{-/-}$and B6.Sst $1^{S}$ lungs segregates from the expression pattern in B6 and $S p 110^{-1-}$ lungs along the PC1 axis (77\% of variance) (Figure 4A). Euclidean distance analysis revealed a similar pattern, with B6.Sst $1^{\mathrm{S}}$ and $S p 140^{-9-}$ mice clustering together, and away from B6 and $S p 110^{-/-}$mice (Figure 4B). At this time point, both $S p 140^{-/-}$and B6.Sst ${ }^{S}$ mice exhibit higher bacterial burdens than B6 and $S p 110^{--}$mice (Figure $2 \mathrm{C}$ ). Thus, the similarity of the gene expression profiles of B6.Sst $1^{S}$ and $S p 140^{-/-}$lungs may merely reflect increased inflammation in these lungs. Alternatively, the increased bacterial burdens may be due to a similarly enhanced type I IFN response in these mice, which leads to secondary bacterial outgrowth and inflammation. Therefore, we specifically compared the change in expression of two subsets of genes: (1) hallmark inflammatory response pathway (Figure 4C) and (2) type I interferon response genes (Figure 4D). This analysis revealed that B6.Sst $1^{S}$ and $S p 140^{-/-}$mice not only show a similarly increased inflammatory gene signature, as expected, but in addition showed a similarly increased type I IFN gene signature. Only 269 genes were significantly differentially expressed (adjusted p-value $<0.05$ ) between $S p 140^{-/-}$and B6.Sst $1^{S}$ samples, whereas 1520 genes were significantly differentially expressed between $S p 140^{-/-}$and B6. Within the 269 genes differentially expressed between $S_{p} 140^{-/-}$and B6.Sst $1^{S}$, 62 were immunoglobulin genes and 62 were annotated as pseudogenes and most differences are only of modest significance (Figure 4E). At present, we cannot explain these differences, but since these genes are not linked to the Sst 1 locus, and since $S p 110^{-1-}$ mice did not exhibit similar changes, we suspect these expression differences reflect background differences between the $S s t 1^{S}$ and $S p 140^{-1-}$ strains. Interestingly, the gene most significantly differentially expressed between B6.Sst $1^{S}$ and $S p 140^{-1-}$ mice (i.e., with the smallest adjusted $p$-value) was $S p 110$ (Figure 4E). This result is expected, given that $S p 110$ is not expressed in B6.Sst ${ }^{S}$ but is retained in our $S p 140^{-}$ mice (Figure S2C). Together, these results show that while they are not identical, the 

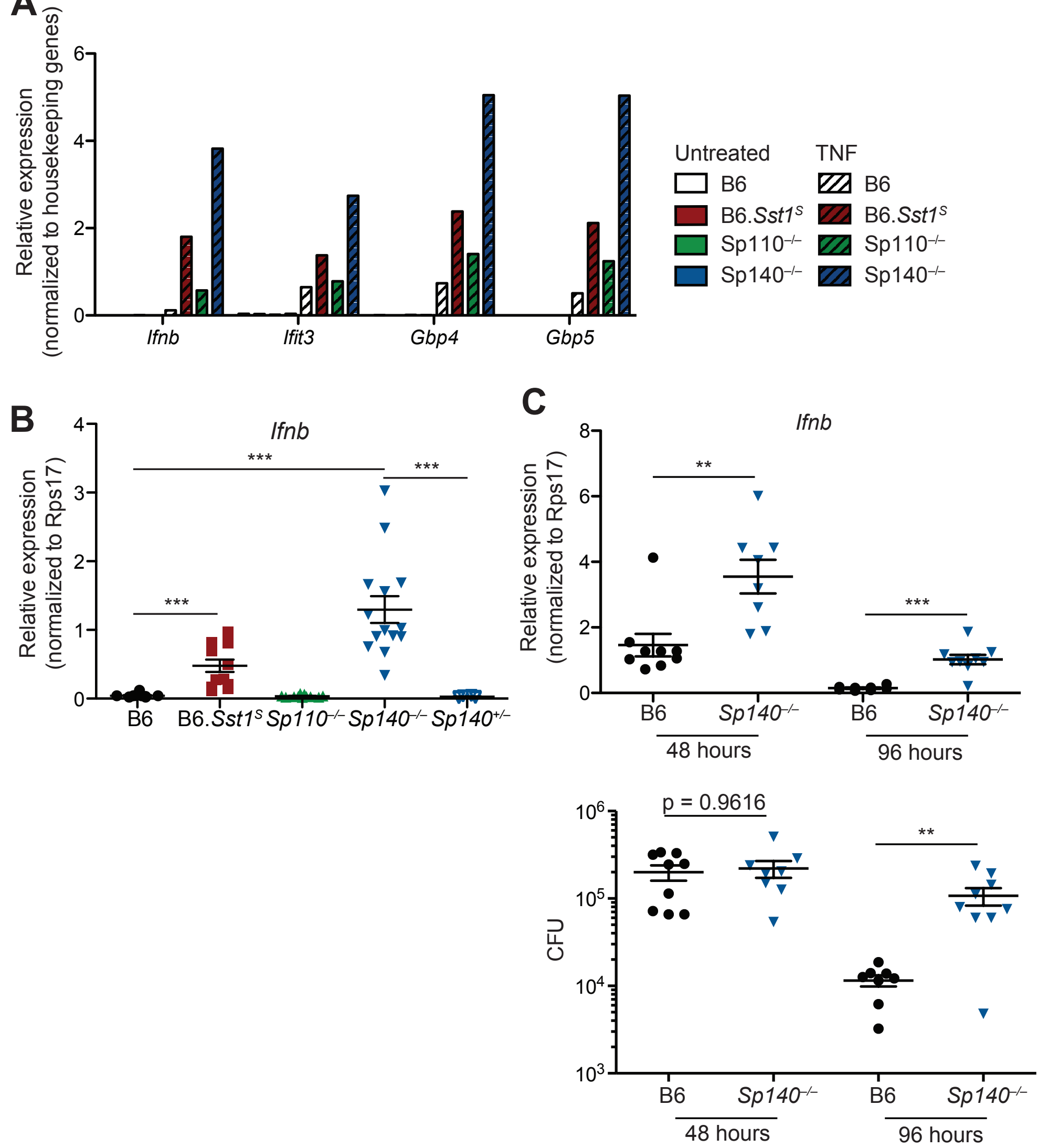

Figure 3. Sp140 $10^{--}$have elevated Ifnb and ISG transcripts. (A) BMMs were left untreated or treated with TNFa for 8 hours. Total RNA were used for RT-qPCR. Representative results of 3 independent experiments. (B) Mice were infected with $M$. tuberculosis and at 28 days post-infection lungs were processed for total RNA, which were used for RT-qPCR. Combined results of 2 independent experiments. (C) Mice were infected with L. pneumophila and RT-qPCR was performed on lungs collected at indicated times. Combined results of 2 independent infections. All mice were bred in-house, $\mathrm{Sp} 14 \mathrm{O}^{--}$and Sp $140^{+/-}$were littermates. (B-C) Mann-Whitney test. * $p \leq 0.05 ;{ }^{* *}, p \leq 0.01 ;{ }^{* *}, p \leq 0.005$. 
bioRxiv preprint doi: https://doi.org/10.1101/2020.01.07.897553; this version posted January 8, 2020. The copyright holder for this preprint (which was not certified by peer review) is the author/funder, who has granted bioRxiv a license to display the preprint in perpetuity. It is made $\triangle 10$. available under aCC-BY-NC 4.0 International license.

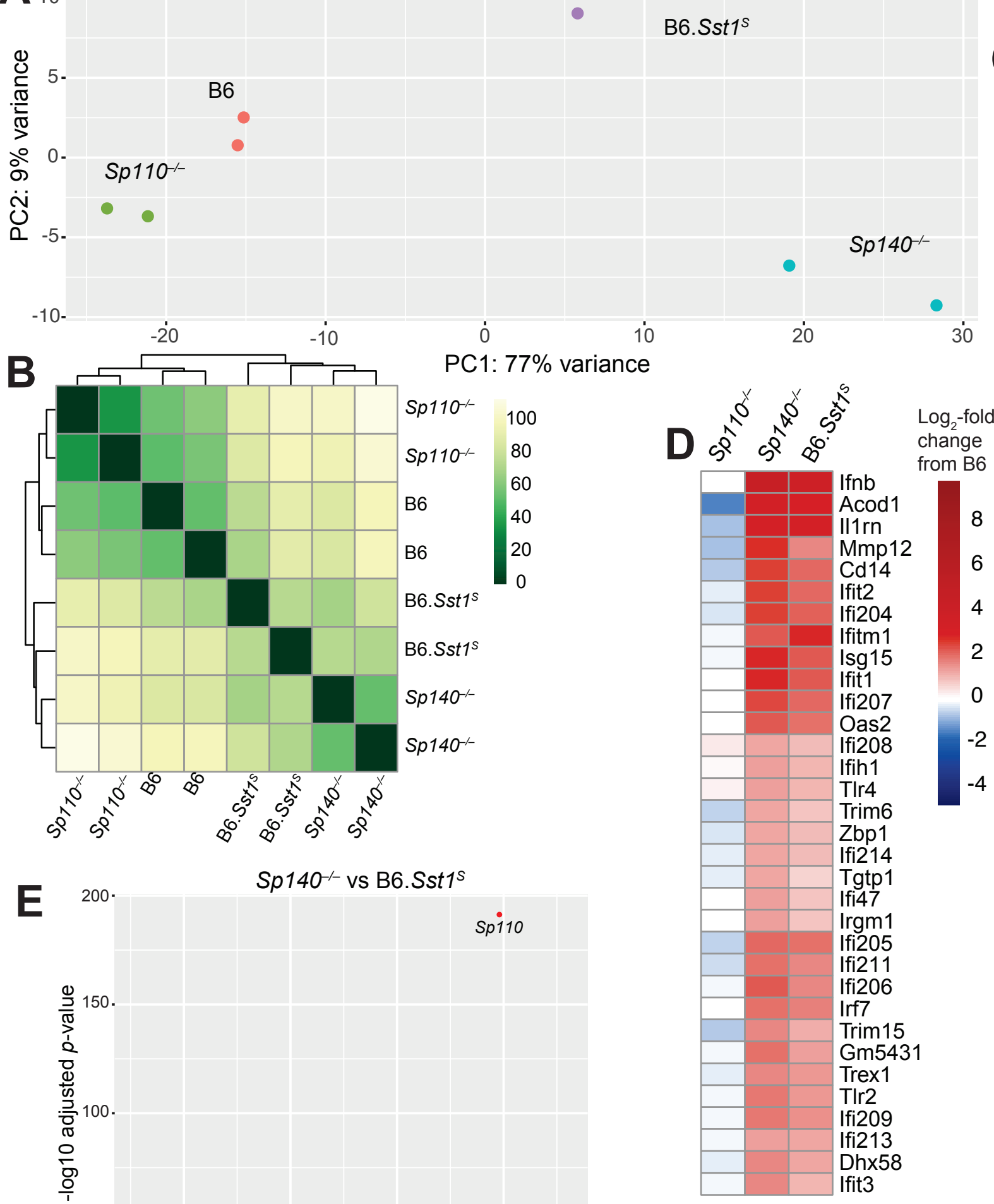

50 .

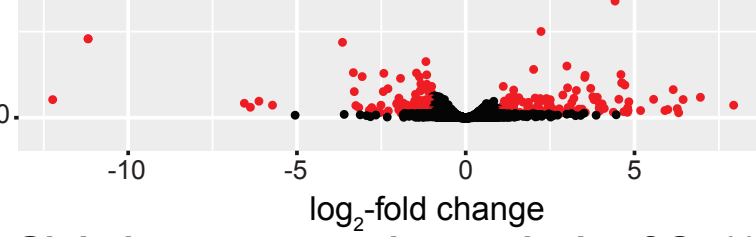

C
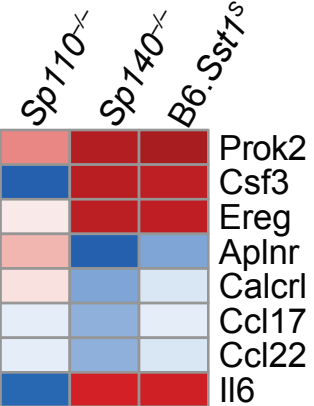

116

II1a

Inhba

Mxd1

Timp1

C5ar1

NIrp3

Serpine1

$\mathrm{Ccl} 2$

Ccl7

Cd14

Ptafr

II $1 \mathrm{~b}$

Osm

C3ar1

Msr1

II7r

Mefv

Cxcl10

Rgs16

Cxcl11

Has2

Marco

Hif1a

Nampt

Tnfrsf9

Clec5a

Rgs1

Sele

Tnfaip6

Csf3r

Plaur

Irf7

Csf1

Itga5

Abca1

Tnfrsf1b

Adora2b

P2ry2

Ptger2

Slc31a2

Gch1

Cd69

Slc28a2

Ccrl2

II15ra

TIr2

F3

Nfkbia

Sgms2

Cdkn1a

Irak2

II10

Osmr

Pcdh7

Sphk1

Bdkrb1

Tpbg

and (D) GO type I IFN response genes. (E) Volcano plot comparing Sp $140^{--}$to B6.Sst ${ }^{\mathrm{S}}$

expression. Dots in red are 2-fold differentially expressed with adjusted $p$-value $\leq 0.05$. 
transcriptomes of $S p 140^{-/}$and B6.Sst $1^{S}$ mice greatly overlap during M. tuberculosis infection, and importantly, both strains exhibit a similar type I IFN signature. Thus it appears that $S p 140^{-1}$ and B6.Sst $1^{S}$ mice may have similar mechanisms of susceptibility to bacterial infection.

Susceptibility of $\mathrm{Sp} 140^{-/-}$mice to bacterial infections is dependent on type I IFN signaling. To determine whether type I IFNs exacerbate $M$. tuberculosis infection of $S p 140^{-1-}$ mice, $M$. tuberculosis-infected $S p 140^{--}$mice were treated with a blocking antibody against IFNAR1. Compared to those mice that only received isotype control antibody, $S p 140^{-1-}$ mice that received the anti-IFNAR1 antibody had reduced bacterial burdens in their lungs (Figure 5A). We also generated Sp $140^{-/-}$Ifnar $^{-\gamma-}$ double-deficient mice and infected them with $M$. tuberculosis (Figure 5B-C). Loss of Ifnar protected Sp $140^{-1-}$ mice from weight loss (Figure 5B) and reduced bacterial burdens at day 25 post-infection, similar to those seen in B6 mice (Figure 5C). Furthermore, Sp $140^{-1}$ Ifnar ${ }^{--}$mice were partially protected from L. pneumophila infection, to a similar degree as B6.Sst $1^{S}$ Ifnar ${ }^{-1}$ mice (Figure 5D-E). These results show that similar to B6.Sst1 $1^{S}$ mice, type I IFN signaling is responsible for the susceptibility of $S p 140^{-/-}$mice to M. tuberculosis, and partially responsible for the susceptibility of $S p 140^{---}$mice to L. pneumophila.

\section{Discussion}

Humans and other vertebrates encounter diverse classes of pathogens, including viruses, bacteria, fungi and parasites. In response, vertebrate immune systems have evolved stereotypical responses appropriate for distinct pathogen types. For example, type I IFN-driven immunity is generally critical for defense against viruses (Schneider et al., 2014; Stetson and Medzhitov, 2006), whereas type II IFN (IFN- $\gamma$ )-driven immunity mediates resistance to intracellular pathogens (Crisler and Lenz, 2018). Additionally, IL-1 is important for inducing neutrophil and other responses against extracellular pathogens (Mantovani et al., 2019), and IL-4/-13 (Type 2 immunity) orchestrates responses to helminths and other parasites (Locksley, 1994). Thus, an important question is how the immune system generates responses that are appropriate for resistance to a specific pathogen while repressing inappropriate responses. The alternative strategy of making all types of responses to all pathogens appears not to be employed, possibly because it would be too energetically costly, or incur too much inflammatory damage to the host. Although there is still much to be learned, it appears that negative feedback is essential to enforce choices between possible types of immune responses. For example, IL-4 and IFN- $\gamma$ have long been appreciated to act as reciprocal negative antagonists of each other (Locksley, 1994). In addition, anti-viral type I IFNs have long been appreciated to negatively regulate IFN- $\gamma$ and IL-1driven anti-bacterial responses (Donovan et al., 2017; Moreira-Teixeira et al., 2018). Although negative regulation of IFN- $\gamma / \mathrm{IL}-1$ by type I IFN is likely beneficial to limit immunopathology during viral infections, $S s t 1^{S}$ mice provide an example of how excessive or inappropriate negative regulation by type I IFN can also be detrimental during bacterial infections (He et al., 2013; Ji et al., 2019). In this study, we therefore sought to understand the molecular mechanisms by which wild-type $\left(S s t 1^{R}\right)$ mice are able to appropriately restrain type I IFNs during bacterial infections.

Although the Sst1 locus was first described in 2005 (Pan et al., 2005), further genetic analysis of the locus has been hindered by its extreme repetitiveness and the concomitant difficulty in generating specific loss-of-function mutations in Sst1-linked genes. In particular, the loss of Sp110 (Iprl) has long been proposed to explain the susceptibility of Sst 1 mice to bacterial infections. However, while we could confirm the loss of Sp110 expression in $S s t 1^{S}$ mice, $S p 110^{-}$ mice were never generated and thus its essential role in host defense has been unclear. The 

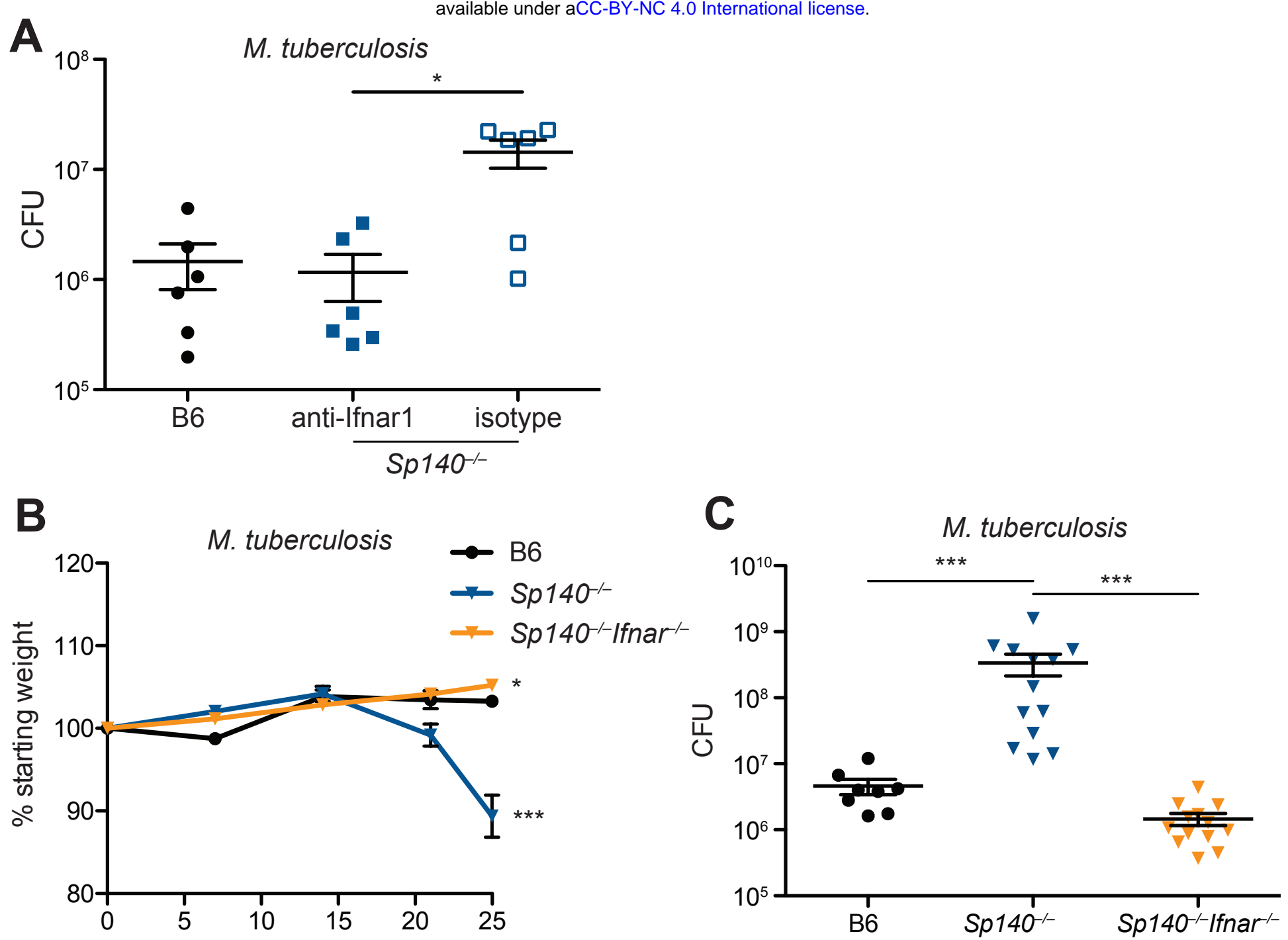

Days post infection

D

L. pneumophila
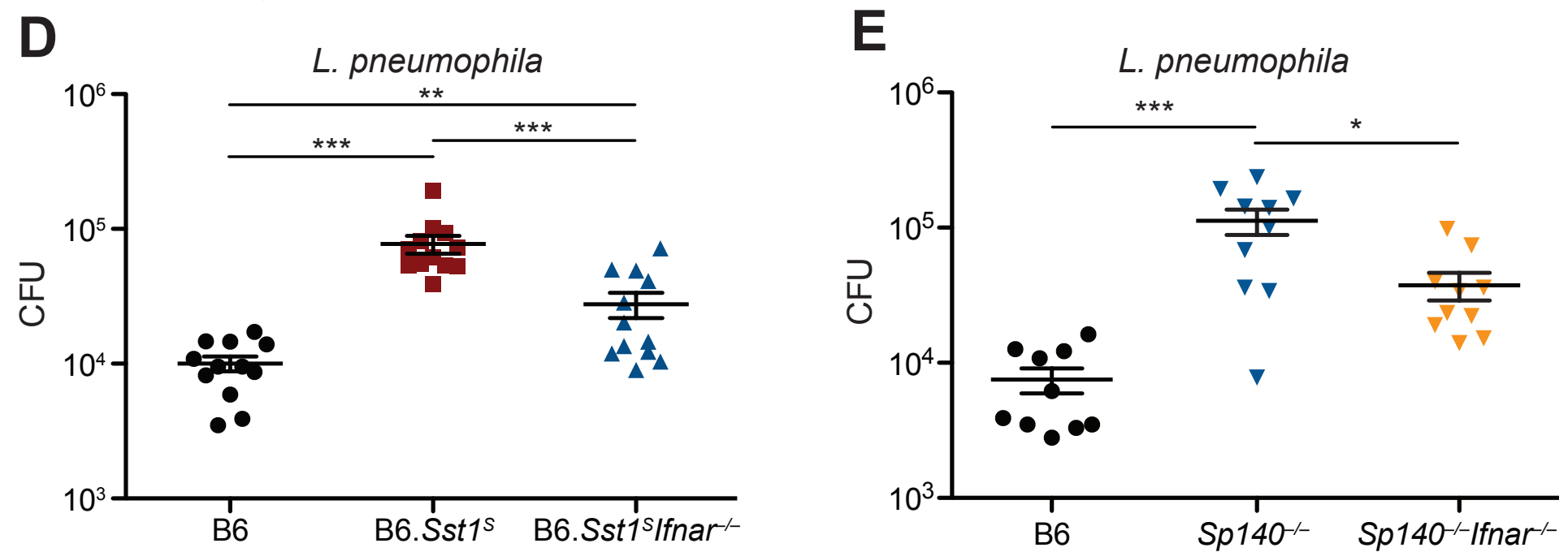

Figure 5. Susceptibility of Sp140-- to M. tuberculosis and L. pneumophila is dependent on type I IFN signaling. (A) Mice were infected with $M$. tuberculosis and treated with either IFNAR1-blocking antibody or isotype control every other day starting 7 days post-infection. At 25 days post-infection lungs were harvested to enumerate CFU. (B-C) Mice were infected with $M$. tuberculosis and measured for body weight (B) and bacteria burdens at day 25 (C). Statistics in B show comparison to B6. Combined results of 2 experiments. (D-E) bacteria burden in L. pneumophila-infected mice at 96 hours. Combined results of 2 experiments. All mice were bred in-house (A-C, E); all but B6 were bred in-house (D). Whitney-Mann test (A-E). ${ }^{*}, p \leq 0.05 ;{ }^{* *}, p \leq 0.01 ;{ }^{* * *}, p \leq 0.005$. 
advent of CRIPSR/Cas9-based methods of genome engineering allowed us to generate $\mathrm{Sp} 110^{-1-}$ mice. Unexpectedly, we found $S p 110^{---}$mice were fully resistant to M. tuberculosis infection, and we thus conclude that lack of $S p 110$ is not sufficient to explain the $S s t 1^{S}$ phenotype. An important caveat of genetic studies of the Sst1 locus is that generating specific gene knockouts is still nearly impossible in this genetic region, even with CRISPR. Indeed, the guide sequence used to target exon 4 of Sp110 also targets an unknown number of pseudogene copies of Sp110like genes located within the unassembled adjacent 'homogenously staining region' of mouse chromosome 1 . Thus, we expect that additional off-target mutations are likely present in our Sp $110^{-1-}$ mutant mice. However, given that the Sp110 pseudogenes are not known to be expressed, we consider it unlikely that collateral mutations would affect our conclusions. Moreover, any off-target mutations should differ among the three founder mice we analyzed and are thus unlikely to explain the consistent resistant phenotype we observed in all three founders. Lastly, since we were able to establish that all the founders at a minimum lack SP110 protein, additional mutations would not affect our conclusion that $S p 110$ is not essential for resistance to M. tuberculosis.

Given that loss of $S p 110$ was not sufficient to explain the susceptibility of $S s t 1^{S}$ mice to bacterial infections, we considered other explanations. We found that $S s t 1^{S}$ mice also lack expression of Sp140, an Sst1-linked homolog of Sp110. Our data suggest that deletion of Sp140 is sufficient to recapitulate the full $S s t 1^{S}$ phenotype including broad susceptibility to multiple bacterial infections including M. tuberculosis, L. monocytogenes, and L. pneumophila. Importantly, the susceptibility of $S p 140^{-1-}$ mice to bacterial infection correlates with an exacerbated type I IFN response, as is also the case for $S s t 1^{S}$ mice. Likewise, as with $S s t 1^{S}$ mice, the susceptibility of $S p 140^{-/-}$mice was rescued by deletion of the gene encoding type I IFN receptor (Ifnar). We therefore conclude that loss of $S p 140$ likely explains the Sst 1 phenotype. It remains possible that the additional loss of $S p 110$ in $S s t 1^{S}$ mice further exacerbates the $S s t 1^{S}$ phenotype as compared to $S p 140^{-1-}$ mice. However, in our studies, we did not observe a consistent difference between $S s t 1^{S}$ (i.e., $S p 110^{-/-} S p 140^{-1-}$ ) mice as compared to our $S p 140^{-/-}$ mice. Another important caveat to our study is that it remains possible that our $S p 140^{-1-}$ mice carry additional mutations, e.g., in Sp140-like genes, that contribute to, or even fully explain, their observed phenotype. This concern is somewhat ameliorated by our analysis of two independent $S p 140^{--}$founders, both of which exhibited susceptibility to M. tuberculosis (Figure $\mathrm{S} 2 \mathrm{E}$ ). In addition, we were able to confirm normal SP110 protein levels in the spleen of uninfected $S p 140^{-1-}$ mice. Furthermore, we observed normal levels of $S p 110$ and $S p 100$ expression at the mRNA level in the lungs of M. tuberculosis-infected mice. Thus, collateral loss of SP100 or SP110 is unlikely to explain the phenotype of our Sp140 ${ }^{-1-}$ mice. Lastly, Sp110 and Sp140 are the only two Sst1-linked genes that we were able to find to be differentially expressed between B6 and B6.Sst $1^{\mathrm{S}}$ mice, and as discussed above, our genetic studies suggest little role for the loss of Sp110. Thus, taken together, our results strongly suggest that loss of SP140 explains the phenotype of our $S p 140^{-1-}$ mice, though we expect that future mechanistic studies will be critical to further test this conclusion.

Because $S p 140$ is inducible by IFN- $\gamma$, our results suggest the existence of a novel feedback loop by which IFN- $\gamma$ acts to repress the transcription of type I IFNs via SP140. This feedback loop appears to be essential for host defense against diverse bacterial pathogens. A major question that remains is how SP140 acts to repress the transcription of type I IFN-induced genes. SP140 contains DNA/histone-binding domains, such as SAND, PHD and Bromodomains, which suggests the hypothesis that SP140 functions as a direct transcriptional repressor of type I IFN genes. However, much more indirect mechanisms are also possible. Interestingly, mouse SP140 localizes to nuclear structures called PML bodies (Bloch et al., 1999). PML bodies are 
implicated in a variety of cell processes such as apoptosis, cell cycle, DNA damage response, senescence, and cell-intrinsic antiviral responses (Scherer and Stamminger, 2016). Whether or not the repressive effects of SP140 on type I IFN expression occur via the activity of PML bodies is an important outstanding question. Another major question is whether or how the repression of type I IFNs by SP140 is specific for bacterial infections and, if not, whether the presence of SP140 impairs anti-viral immunity. Lastly, polymorphisms in human SP140 are associated with chronic lymphocytic leukemia (CLL), Crohn's disease, and multiple sclerosis (MS) (Franke et al., 2010; Jostins et al., 2012; Karaky et al., 2018; Matesanz et al., 2015; Slager et al., 2013). Studies using siRNA and shRNA-mediated knockdown have also implicated SP140 in the repression of lineage-inappropriate genes in macrophages (Mehta et al., 2017). Our generation of $S p 140^{--}$mice is therefore important to permit future studies into these alternative roles of SP140.

\section{Methods}

Mice. All mice were specific pathogen-free, maintained under a 12-hr light-dark cycle (7AM to 7PM), and given a standard chow diet (Harlan irradiated laboratory animal diet) ad libitum. All mice were sex and age-matched at 6-10 weeks old at the beginning of infections. C57BL/6J (B6) and $\mathrm{B} 6(\mathrm{Cg})$-Ifnarltm 1.2Ees/J (Ifnar $\left.{ }^{-1}\right)$ were originally purchased from Jackson Laboratories and subsequently bred at UC Berkeley. B6J.C3-Sst ${ }^{\mathrm{C} 3 \mathrm{HeB} / \mathrm{FeJ}} \mathrm{Krmn}$ mice (referred to as B6.Sst ${ }^{\mathrm{S}}$ throughout) were from the colony of I. Kramnik at Boston University and then transferred to UC Berkeley. CRISPR/Cas9 targeting was performed by pronuclear injection of Cas9 mRNA and sgRNA into fertilized zygotes from colony-born C57BL/6J mice, essentially as described previously (Wang et al., 2013). Founder mice were genotyped as described below, and founders carrying Sp140 mutations were bred one generation to C57BL/6J to separate modified Sp140 haplotypes. Homozygous lines were generated by interbreeding heterozygotes carrying matched Sp140 haplotypes. Sp140 ${ }^{--}$Ifnar ${ }^{--}$were generated by crossing the Sp140-- and Ifnar ${ }^{--}$mice inhouse. All animals used in experiments were bred in-house unless otherwise noted in the figure legends. All animal experiments complied with the regulatory standards of, and were approved by, the University of California Berkeley Institutional Animal Care and Use Committee.

Genotyping of $S p 140$ alleles. Exon 3 and the surrounding intronic regions were amplified by bracket PCR using the following primers (all 5' to 3'): Sp140-1 fwd, ACGAATAGCAAGCAGGAATGCT, and rev, GGTTCCGGCTGAGCACTTAT. The PCR products are diluted at 1:10 and $2 \mu \mathrm{l}$ were used as template for the second PCR using the following primers: Sp140-2 fwd, TGAGGACAGAACTCAGGGAG, and rev, ACACGCCTTTAATCCCAGCATTT. The primer combinations were designed to distinguish Sp140 from other Sp140-like genes. Primers were used at 200nM in each 20 $\mu 1$ reaction with 1x Dreamtaq Green PCR Master Mix (Thermo Fisher Scientific). Cleaned PCR products were diluted at 1:10 and sequenced using Sanger sequencing (Elim Biopharm).

Mycobacterium tuberculosis infections. M. tuberculosis strain Erdman (gift of S.A. Stanley) was used for all infections. Frozen stocks of this wild-type strain were made from a single culture and used for all experiments. Cultures for infection were grown in Middlebrook 7H9 liquid medium supplemented with $10 \%$ albumin-dextrose-saline, $0.4 \%$ glycerol and $0.05 \%$ Tween-80 for five days at $37^{\circ} \mathrm{C}$. Mice were aerosol infected using an inhalation exposure system (Glas-Col, Terre Haute, IN). A total of $9 \mathrm{ml}$ of diluted culture was loaded into the nebulizer calibrated to deliver $\sim 20$ to 50 bacteria per mouse as confirmed by measurement of colony forming units (CFUs) in the lungs 1 day following infection. Mice were sacrificed at various 
days post-infection (as described in figure legends) to measure CFUs and RNA levels. All but one lung lobe was homogenized in PBS plus $0.05 \%$ Tween- 80 , and serial dilutions were plated on $7 \mathrm{H} 11$ plates supplemented with $10 \%$ oleic acid, albumin, dextrose, catalase (OADC) and $0.5 \%$ glycerol. CFUs were counted 21 days after plating. The remaining lobe was used for histology or for RNA extraction. For histology, the sample was fixed in 10\% formalin for at least 48 hours then stored in 70\% ethanol. Samples were sent to Histowiz Inc for embedding in wax, sectioning and staining with hematoxylin and eosin. For survival experiments, mice were monitored for weight loss and were euthanized when they reached a humane endpoint as determined by the University of California Berkeley Institutional Animal Care and Use Committee.

Legionella pneumophila infections. Infections were performed using L. pneumophila strain JR32 $\Delta$ flaA (gift of D.S. Zamboni) as previously described (Goncalves et al., 2019; Mascarenhas et al., 2015). Briefly, frozen cultures were streaked out on to BCYE plates to obtain single colonies. A single colony was chosen and streaked on to a new BCYE plate to obtain a $1 \mathrm{~cm}$ by $1 \mathrm{~cm}$ square bacterial lawn, and incubated for 2 days at $37^{\circ} \mathrm{C}$. The patch was solubilized in autoclaved MilliQ water and the optical density was measured at $600 \mathrm{~nm}$. Culture was diluted to $2.5 \times 10^{6}$ bacteria $/ \mathrm{ml}$ in sterile PBS. The mice were first anesthetized with ketamine and xylazine $(90 \mathrm{mg} / \mathrm{kg}$ and $5 \mathrm{mg} / \mathrm{kg}$, respectively) by intraperitoneal injection then infected intranasally with $40 \mu \mathrm{L}$ with PBS containing a final dilution of $1 \times 10^{5}$ bacteria per mouse. For enumerating of CFU, the lungs were harvested and homogenized in $5 \mathrm{~mL}$ of autoclaved MilliQ water for 30 seconds, using a tissue homogenizer. Lung homogenates were diluted in autoclaved MilliQ water and plated on BCYE agar plates. CFU was enumerated after plates were incubated for 4 days at $37^{\circ} \mathrm{C}$.

Listeria monocytogenes infections. For in vivo infections, bacterial cultures were grown overnight in $\mathrm{BHI}$, diluted 1:5 in $\mathrm{BHI}$ and grown at $37^{\circ} \mathrm{C}$ for $1.5 \mathrm{~h}$ until they reached an optical density at $600 \mathrm{~nm}$ of 0.5 . Mice were injected with $10^{5}$ wildtype (10403S) bacteria intravenously by the tail vein. At $48 \mathrm{~h}$ post-infection, the spleens and livers were harvested, homogenized, and plated in serial dilutions to enumerate CFU.

Bone marrow-derived macrophages (BMMs) and TNF-treatment. Bone marrow was harvested from mouse femurs and tibias, and cells were differentiated by culture on non-tissue culture-treated plates in RPMI supplemented with supernatant from 3T3-MCSF cells (gift of B. Beutler), $10 \%$ fetal bovine serum (FBS), $2 \mathrm{mM}$ glutamine, $100 \mathrm{U} / \mathrm{ml}$ streptomycin and $100 \mu \mathrm{g} / \mathrm{ml}$ penicillin in a humidified incubator $\left(37^{\circ} \mathrm{C}, 5 \% \mathrm{CO} 2\right)$. BMMs were harvested six days after plating and frozen in $95 \% \mathrm{FBS}$ and $5 \%$ DMSO. For in vitro experiments, BMMs were thawed into media as described above for 4 hours in a humidified $37^{\circ} \mathrm{C}$ incubator. Adherent cells were washed with PBS, counted and replated at $1.2 \times 10^{6} \sim 1.5 \times 10^{6}$ cells/well in a TC-treated 6-well plate. Cells were treated with $10 \mathrm{ng} / \mathrm{ml}$ recombinant mouse TNF $\alpha(410-\mathrm{TRNC}-010, \mathrm{R} \& \mathrm{D}$ systems) diluted in the media as described above.

Quantitative/conventional RT-PCR. Total RNA from BMMs was extract using E.Z.N.A. Total RNA Kit I (Omega Bio-tek) according to manufacturer specifications. Total RNA from infected tissues was extracted by homogenizing in TRIzol reagent (Life technologies) then mixing thoroughly with chloroform, both done under BSL3 conditions. Samples were then removed from the BSL3 facility and transferred to fresh tubes under BSL2 conditions. Aqueous phase was separated by centrifugation and RNA was further purified using the E.Z.N.A. Total RNA Kit I (Omega Bio-tek). Equal amounts of RNA from each sample were treated with DNase (RQ1, Promega) and cDNA was made using Superscript III (Invitrogen). Complementary cDNA reactions were primed with poly $(\mathrm{dT})$ for the measurement of mature transcripts. For experiments with multiple time points, macrophage samples were frozen in the RLT buffer (Qiagen) and 
infected tissue samples in RNAlater ${ }^{\mathrm{TM}}$ solution (Invitrogen) and processed to RNA at the same time. Quantitative PCR was performed using QuantiStudio 5 Real-Time PCR System (Applied Biosystems) with Power Sybr Green PCR Master Mix (Thermo Fisher Scientific) according manufacturer specifications. Transcript levels were normalized to housekeeping genes Rps 17, Actb and Oazl unless otherwise specified. The following primers were used in this study. Rps 17 sense: CGCCATTATCCC CAGCAAG; Rps 17 antisense: TGTCGGGATCCACCTCAATG; Oazl sense: GTG GTG GCC TCT ACA TCG AG; Oazl antisense: AGC AGA TGA AAA CGT GGT CAG; $A c t b$ sense: CGC AGC CAC TGT CGA GTC; $A c t b$ antisense: CCT TCT GAC CCA TTC CCA CC; Ifnb sense: GTCCTCAACTGCTCTCCACT; Ifnb antisense: CCTGCAACCACCACTCATTC; Gbp 4 sense: TGAGTACCTGGAGAATGCCCT; Gbp 4 antisense: TGGCCGAATTGGATGCTTGG; Gbp5 sense: TGTTCTTACTGGCCCCTGCT; Gbp5 antisense: CCAATGAGGCACAAGGGTTC; Ifit3 sense: AGCCCACACCCAGCTTTT; Ifit3 antisense: CAGAGATTCCCGGTTGACCT. Conventional RT-PCR shown in Figure 2A was done using the following primers. Sense: GTCCCTTGGAGTCTGTGTAGG; antisense: CATCCTGGGGCTCTTGTCTTG. Primers were used at $200 \mathrm{nM}$ in each $20 \mu 1$ reaction with $1 \mathrm{x}$ Dreamtaq Green PCR Master Mix (Thermo Fisher Scientific).

Immunoblot. Samples were lysed in RIPA buffer with protease inhibitor cocktail (Roche) to obtain total protein lysate and were clarified by spinning at $\sim 16,000 \times \mathrm{g}$ for $30 \mathrm{~min}$ at $4{ }^{\circ} \mathrm{C}$. Clarified lysates were analyzed with Pierce BCA protein assay kit (Thermo Fisher Scientific) according to manufacturer specification and diluted to the same concentration and denatured with SDS-loading buffer. Samples were separated on NuPAGE Bis-Tris $4 \%$ to $12 \%$ gradient gels (Thermo Fisher Scientific) following the manufacturer's protocol. Gels were transferred onto ImmobilonFL PVDF membranes at $35 \mathrm{~V}$ for 90 min and blocked with Odyssey blocking buffer (Li-Cor). Proteins were detected on a Li-Cor Odyssey Blot Imager using the following primary and secondary antibodies. Rabbit anti-SP110 or SP140 serums were produced by Covance and used at 1:1000 dilution. Hybridoma cells expressing monoclonal anti-SP110 antibody were a gift of I. Kramnik. Antibodies were produced in-house as previously described (Ji et al., 2019) and used at 100ng/ml. Alexa Fluor 680-conjugated secondary antibodies (Invitrogen) were used at $0.4 \mathrm{mg} / \mathrm{ml}$.

RNA sequencing and analysis. Total RNA was isolated as described above. Illuminacompatible libraries were generated by the University of California, Berkeley, QB3 Vincent J. Coates Genomics Sequencing Laboratory. The libraries were multiplexed and sequenced using one flow cell on Novaseq 6000 (Illumina) as 50bp paired-end reads. The data were aligned using Sleuth (Pimentel et al., 2017) and analyzed using DEseq2 (Love et al., 2014) and DEVis packages (Price et al., 2019).

Antibody-mediated neutralization. For all antibody treatments, the schedules are indicated in the figures. All treatments were delivered by intraperitoneal injection. Mouse anti-mouse IFNAR1 (MAR1-5A3) and isotype control (GIR208, mouse anti-human IFNGR- $\alpha$ chain) were purchased from Leinco Technologies Inc. For injections antibody stocks were diluted in sterile

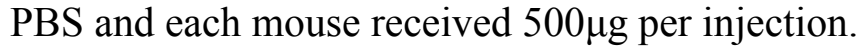

Statistical analysis. All data were analyzed with Mann-Whitney test unless otherwise noted. Tests were run using GraphPad Prism 5. *, $p \leq 0.05 ; * *, p \leq 0.01 ; * * *, p \leq 0.001$. All error bars are s.e. 
Acknowledgements: We thank the Stanley and Cox laboratories for discussions and for support with M. tuberculosis experiments, L. Flores, P. Dietzen and R. Chavez for technical assistance, and members of the Vance, Barton, Cox, Stanley, and Portnoy labs for advice and discussions.

Author contributions: Conceptualization-D.X.J., K.H.D., A.Y.L., R.E.V.; MethodologyD.X.J., K.C.W., D.I.K., S.R.M., A.L., K.J.C., A.Y.L., D.S.Z.; Validation-XXX; Resources-I.K., D.A.P., R.E.V.; Data curation-D.X.J.; Writing - original draft preparation-D.X.J., R.E.V.; Writing - review \& editing-D.X.J., K.C.W., D.I.K., K.H.D., R.E.V.; Supervision-D.A.P., K.H.D., R.E.V.; Funding acquisition-D.A.P., K.H.D., R.E.V.

Funding: R.E.V. is supported by an Investigator Award from the Howard Hughes Medical Institute. This work was also supported by NIH grants R37AI075039 (R.E.V.), P01AI066302 (R.E.V. and D.A.P.). R.E.V. and K.H.D. were Burroughs Wellcome Fund Investigators in the Pathogenesis of Infectious Disease.

Competing Interests: R.E.V.'s spouse is an employee of Aduro Biotech, a company that is developing STING agonists to induce type I interferons for cancer immunotherapy. R.E.V. may therefore benefit from the commercialization of the results of this research.

Ethics Statement: Animal studies were approved by the UC Berkeley Animal Care and Use Committee.

\section{References}

Anderson, M.S., and Su, M.A. (2016). AIRE expands: new roles in immune tolerance and beyond. Nature reviews Immunology 16, 247-258.

Auerbuch, V., Brockstedt, D.G., Meyer-Morse, N., O'Riordan, M., and Portnoy, D.A. (2004). Mice lacking the type I interferon receptor are resistant to Listeria monocytogenes. The Journal of experimental medicine 200, 527-533.

Bhattacharya, B., Chattrerjee, S., Berland, R., Pichugin, A.V., Gao, Y., Connor, J., Ivanov, A., Yan, B.S., Kobzik, L., and Kramnik, I. (2018). Increased susceptibility to intracellular bacteria and necrotic inflammation driven by a dysregulated macrophage response to TNF. bioRxiv.

Bloch, D.B., Chiche, J.D., Orth, D., de la Monte, S.M., Rosenzweig, A., and Bloch, K.D. (1999). Structural and functional heterogeneity of nuclear bodies. Molecular and cellular biology 19, 4423-4430.

Bottomley, M.J., Collard, M.W., Huggenvik, J.I., Liu, Z., Gibson, T.J., and Sattler, M. (2001). The SAND domain structure defines a novel DNA-binding fold in transcriptional regulation. Nature structural biology 8, 626-633.

Boxx, G.M., and Cheng, G. (2016). The Roles of Type I Interferon in Bacterial Infection. Cell host \& microbe 19, 760-769.

Boyartchuk, V., Rojas, M., Yan, B.S., Jobe, O., Hurt, N., Dorfman, D.M., Higgins, D.E., Dietrich, W.F., and Kramnik, I. (2004). The host resistance locus sst1 controls innate immunity to Listeria monocytogenes infection in immunodeficient mice. Journal of immunology 173, 5112-5120.

Carrero, J.A., Calderon, B., and Unanue, E.R. (2004). Type I interferon sensitizes lymphocytes to apoptosis and reduces resistance to Listeria infection. The Journal of experimental medicine 200, 535-540. 
Chang, S.Y., Chen, M.L., Lee, M.R., Liang, Y.C., Lu, T.P., Wang, J.Y., and Yan, B.S. (2018). SP110 Polymorphisms Are Genetic Markers for Vulnerability to Latent and Active Tuberculosis Infection in Taiwan. Disease markers 2018, 4687380.

Crisler, W.J., and Lenz, L.L. (2018). Crosstalk between type I and II interferons in regulation of myeloid cell responses during bacterial infection. Current opinion in immunology 54, 35-41.

Donovan, M.L., Schultz, T.E., Duke, T.J., and Blumenthal, A. (2017). Type I Interferons in the Pathogenesis of Tuberculosis: Molecular Drivers and Immunological Consequences. Frontiers in immunology 8, 1633.

Dorhoi, A., Yeremeev, V., Nouailles, G., Weiner, J., 3rd, Jorg, S., Heinemann, E., OberbeckMuller, D., Knaul, J.K., Vogelzang, A., Reece, S.T., et al. (2014). Type I IFN signaling triggers immunopathology in tuberculosis-susceptible mice by modulating lung phagocyte dynamics. European journal of immunology 44, 2380-2393.

Fox, G.J., Sy, D.N., Nhung, N.V., Yu, B., Ellis, M.K., Van Hung, N., Cuong, N.K., Thi Lien, L., Marks, G.B., Saunders, B.M., et al. (2014). Polymorphisms of SP110 are associated with both pulmonary and extra-pulmonary tuberculosis among the Vietnamese. PloS one 9, e99496.

Franke, A., McGovern, D.P., Barrett, J.C., Wang, K., Radford-Smith, G.L., Ahmad, T., Lees, C.W., Balschun, T., Lee, J., Roberts, R., et al. (2010). Genome-wide meta-analysis increases to 71 the number of confirmed Crohn's disease susceptibility loci. Nature genetics 42,1118 1125.

Goncalves, A.V., Margolis, S.R., Quirino, G.F.S., Mascarenhas, D.P.A., Rauch, I., Nichols, R.D., Ansaldo, E., Fontana, M.F., Vance, R.E., and Zamboni, D.S. (2019). Gasdermin-D and Caspase-7 are the key Caspase-1/8 substrates downstream of the NAIP5/NLRC4 inflammasome required for restriction of Legionella pneumophila. PLoS pathogens 15 , e1007886.

He, X., Berland, R., Mekasha, S., Christensen, T.G., Alroy, J., Kramnik, I., and Ingalls, R.R. (2013). The sstl resistance locus regulates evasion of type I interferon signaling by Chlamydia pneumoniae as a disease tolerance mechanism. PLoS pathogens 9, e1003569.

Ji, D.X., Yamashiro, L.H., Chen, K.J., Mukaida, N., Kramnik, I., Darwin, K.H., and Vance, R.E. (2019). Type I interferon-driven susceptibility to Mycobacterium tuberculosis is mediated by IL-1Ra. Nature microbiology 4, 2128-2135.

Jostins, L., Ripke, S., Weersma, R.K., Duerr, R.H., McGovern, D.P., Hui, K.Y., Lee, J.C., Schumm, L.P., Sharma, Y., Anderson, C.A., et al. (2012). Host-microbe interactions have shaped the genetic architecture of inflammatory bowel disease. Nature 491, 119-124.

Karaky, M., Fedetz, M., Potenciano, V., Andres-Leon, E., Codina, A.E., Barrionuevo, C., Alcina, A., and Matesanz, F. (2018). SP140 regulates the expression of immune-related genes associated with multiple sclerosis and other autoimmune diseases by NF-kappaB inhibition. Human molecular genetics 27, 4012-4023.

Lei, X., Zhu, H., Zha, L., and Wang, Y. (2012). SP110 gene polymorphisms and tuberculosis susceptibility: a systematic review and meta-analysis based on 10624 subjects. Infection, genetics and evolution : journal of molecular epidemiology and evolutionary genetics in infectious diseases 12, 1473-1480.

Locksley, R.M. (1994). Th2 cells: help for helminths. The Journal of experimental medicine 179, 1405-1407.

Love, M.I., Huber, W., and Anders, S. (2014). Moderated estimation of fold change and dispersion for RNA-seq data with DESeq2. Genome biology 15, 550. 
Mantovani, A., Dinarello, C.A., Molgora, M., and Garlanda, C. (2019). Interleukin-1 and Related Cytokines in the Regulation of Inflammation and Immunity. Immunity 50, 778-795.

Mascarenhas, D.P., Pereira, M.S., Manin, G.Z., Hori, J.I., and Zamboni, D.S. (2015). Interleukin 1 receptor-driven neutrophil recruitment accounts to MyD88-dependent pulmonary clearance of legionella pneumophila infection in vivo. The Journal of infectious diseases 211, 322-330.

Matesanz, F., Potenciano, V., Fedetz, M., Ramos-Mozo, P., Abad-Grau Mdel, M., Karaky, M., Barrionuevo, C., Izquierdo, G., Ruiz-Pena, J.L., Garcia-Sanchez, M.I., et al. (2015). A functional variant that affects exon-skipping and protein expression of SP140 as genetic mechanism predisposing to multiple sclerosis. Human molecular genetics 24, 5619-5627.

Mayer-Barber, K.D., Andrade, B.B., Oland, S.D., Amaral, E.P., Barber, D.L., Gonzales, J., Derrick, S.C., Shi, R., Kumar, N.P., Wei, W., et al. (2014). Host-directed therapy of tuberculosis based on interleukin-1 and type I interferon crosstalk. Nature 511, 99-103.

McNab, F., Mayer-Barber, K., Sher, A., Wack, A., and O'Garra, A. (2015). Type I interferons in infectious disease. Nature reviews Immunology 15, 87-103.

Mehta, S., Cronkite, D.A., Basavappa, M., Saunders, T.L., Adiliaghdam, F., Amatullah, H., Morrison, S.A., Pagan, J.D., Anthony, R.M., Tonnerre, P., et al. (2017). Maintenance of macrophage transcriptional programs and intestinal homeostasis by epigenetic reader SP140. Science immunology 2.

Moreira-Teixeira, L., Mayer-Barber, K., Sher, A., and O'Garra, A. (2018). Type I interferons in tuberculosis: Foe and occasionally friend. The Journal of experimental medicine 215, 1273 1285.

O'Connell, R.M., Saha, S.K., Vaidya, S.A., Bruhn, K.W., Miranda, G.A., Zarnegar, B., Perry, A.K., Nguyen, B.O., Lane, T.F., Taniguchi, T., et al. (2004). Type I interferon production enhances susceptibility to Listeria monocytogenes infection. The Journal of experimental medicine 200, 437-445.

Pan, H., Yan, B.S., Rojas, M., Shebzukhov, Y.V., Zhou, H., Kobzik, L., Higgins, D.E., Daly, M.J., Bloom, B.R., and Kramnik, I. (2005). Ipr1 gene mediates innate immunity to tuberculosis. Nature 434, 767-772.

Perniola, R., and Musco, G. (2014). The biophysical and biochemical properties of the autoimmune regulator (AIRE) protein. Biochimica et biophysica acta 1842, 326-337.

Pichugin, A.V., Yan, B.S., Sloutsky, A., Kobzik, L., and Kramnik, I. (2009). Dominant role of the sst1 locus in pathogenesis of necrotizing lung granulomas during chronic tuberculosis infection and reactivation in genetically resistant hosts. The American journal of pathology 174, 2190-2201.

Pilla-Moffett, D., Barber, M.F., Taylor, G.A., and Coers, J. (2016). Interferon-Inducible GTPases in Host Resistance, Inflammation and Disease. Journal of molecular biology 428, 3495-3513.

Pimentel, H., Bray, N.L., Puente, S., Melsted, P., and Pachter, L. (2017). Differential analysis of RNA-seq incorporating quantification uncertainty. Nature methods 14, 687-690.

Png, E., Alisjahbana, B., Sahiratmadja, E., Marzuki, S., Nelwan, R., Adnan, I., van de Vosse, E., Hibberd, M., van Crevel, R., Ottenhoff, T.H., et al. (2012). Polymorphisms in SP110 are not associated with pulmonary tuberculosis in Indonesians. Infection, genetics and evolution : journal of molecular epidemiology and evolutionary genetics in infectious diseases 12,1319 1323.

Price, A., Caciula, A., Guo, C., Lee, B., Morrison, J., Rasmussen, A., Lipkin, W.I., and Jain, K. (2019). DEvis: an R package for aggregation and visualization of differential expression data. BMC bioinformatics 20, 110 . 
Rayamajhi, M., Humann, J., Penheiter, K., Andreasen, K., and Lenz, L.L. (2010). Induction of IFN-alphabeta enables Listeria monocytogenes to suppress macrophage activation by IFNgamma. The Journal of experimental medicine 207, 327-337.

Roscioli, T., Cliffe, S.T., Bloch, D.B., Bell, C.G., Mullan, G., Taylor, P.J., Sarris, M., Wang, J., Donald, J.A., Kirk, E.P., et al. (2006). Mutations in the gene encoding the PML nuclear body protein Sp1 10 are associated with immunodeficiency and hepatic veno-occlusive disease. Nature genetics 38, 620-622.

Scherer, M., and Stamminger, T. (2016). Emerging Role of PML Nuclear Bodies in Innate Immune Signaling. Journal of virology 90, 5850-5854.

Schneider, W.M., Chevillotte, M.D., and Rice, C.M. (2014). Interferon-stimulated genes: a complex web of host defenses. Annual review of immunology 32, 513-545.

Slager, S.L., Caporaso, N.E., de Sanjose, S., and Goldin, L.R. (2013). Genetic susceptibility to chronic lymphocytic leukemia. Seminars in hematology 50, 296-302.

Stanley, S.A., Johndrow, J.E., Manzanillo, P., and Cox, J.S. (2007). The Type I IFN response to infection with Mycobacterium tuberculosis requires ESX-1-mediated secretion and contributes to pathogenesis. Journal of immunology 178, 3143-3152.

Stetson, D.B., and Medzhitov, R. (2006). Type I interferons in host defense. Immunity 25, 373381.

Thye, T., Browne, E.N., Chinbuah, M.A., Gyapong, J., Osei, I., Owusu-Dabo, E., Niemann, S., Rusch-Gerdes, S., Horstmann, R.D., and Meyer, C.G. (2006). No associations of human pulmonary tuberculosis with Sp110 variants. Journal of medical genetics 43, e32.

Tosh, K., Campbell, S.J., Fielding, K., Sillah, J., Bah, B., Gustafson, P., Manneh, K., Lisse, I., Sirugo, G., Bennett, S., et al. (2006). Variants in the SP110 gene are associated with genetic susceptibility to tuberculosis in West Africa. Proceedings of the National Academy of Sciences of the United States of America 103, 10364-10368.

Wang, H., Yang, H., Shivalila, C.S., Dawlaty, M.M., Cheng, A.W., Zhang, F., and Jaenisch, R. (2013). One-step generation of mice carrying mutations in multiple genes by CRISPR/Casmediated genome engineering. Cell 153, 910-918.

Weichenhan, D., Kunze, B., Winking, H., van Geel, M., Osoegawa, K., de Jong, P.J., and Traut, W. (2001). Source and component genes of a 6-200 Mb gene cluster in the house mouse. Mammalian genome : official journal of the International Mammalian Genome Society 12, 590-594.

Zhang, S., Wang, X.B., Han, Y.D., Wang, C., Zhou, Y., and Zheng, F. (2017). Certain Polymorphisms in SP110 Gene Confer Susceptibility to Tuberculosis: A Comprehensive Review and Updated Meta-Analysis. Yonsei medical journal 58, 165-173. 
bioRxiv preprint doi: https://doi.org/10.1101/2020.01.07.897553; this version posted January 8, 2020. The copyright holder for this preprint (which was not certified by peer review) is the author/funder, who has granted bioRxiv a license to display the preprint in perpetuity. It is made

A available under aCC-BY-NC 4.0 International license.

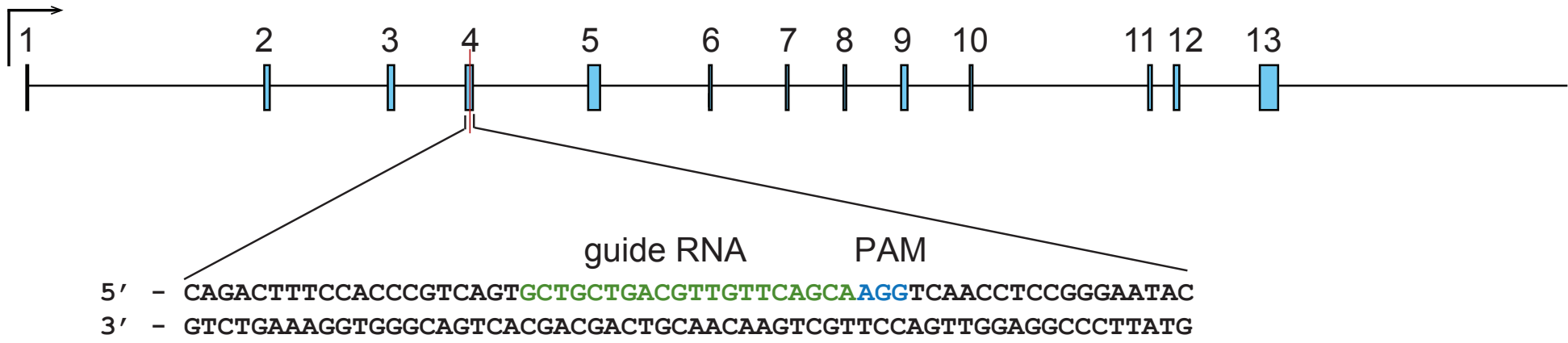

B

D

Founder 61
5 bp deletion

WT GCTGCTGACGITGTTMAGCAAGGTCAA

Founder 65

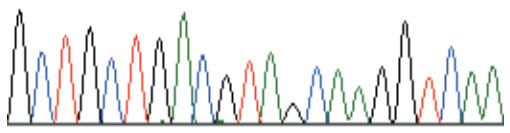

C

Founder 71
$1 \mathrm{bp}$ insertion

WT

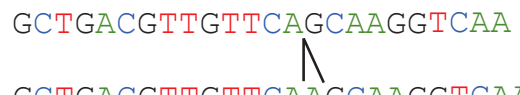

GCTGACGTTGTTCAAGCAAGGTCAA

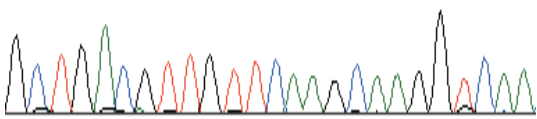

307bp insertion

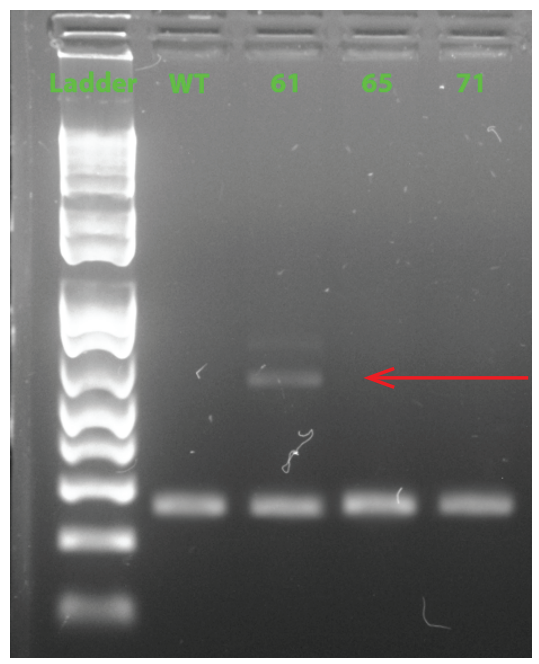

Figure S1. CRISPR/Cas9 targeting strategy for Sp110-- mice. (A) Mouse Sp110 gene. Guide RNA sequence for CRISPR/Cas9 targeting and protospacer-adjacent motif (PAM) are indicated. (B-D) Sp110 locus in wildtype (WT) and three independent lines. Homozygotes of 2 lines identified by sequencing (B-C), and heterozygote of the 3rd line by PCR products separated on an agarose gel (D). Arrow indicates the mutant band. 


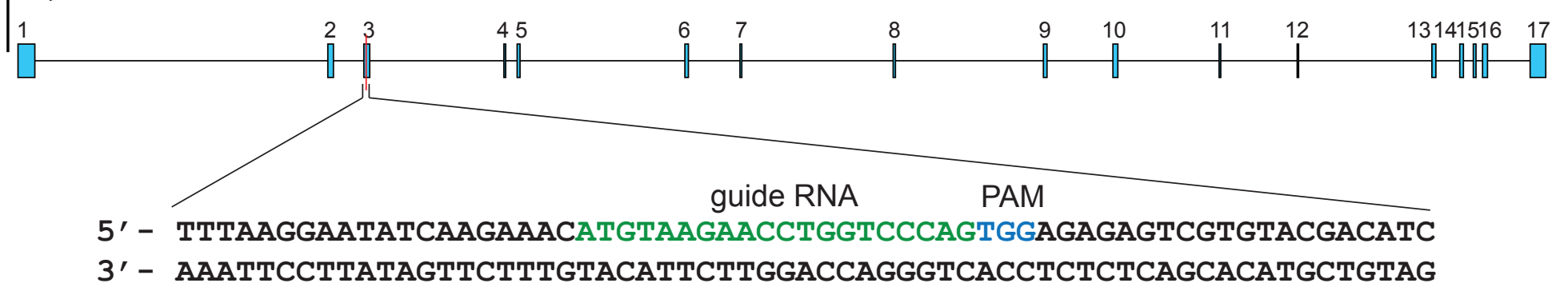

B

WT AGAACCTGGTCCCAGTGGAGAGA Sp140-^1 AGAACCTGGTCAGTGGAGAGA whom hom was

C WT AGAACCTGGTCCCAGTGGAGAGA Sp 140- 2 AGAACCTGGTCCAGTGGAGAGA summonanow

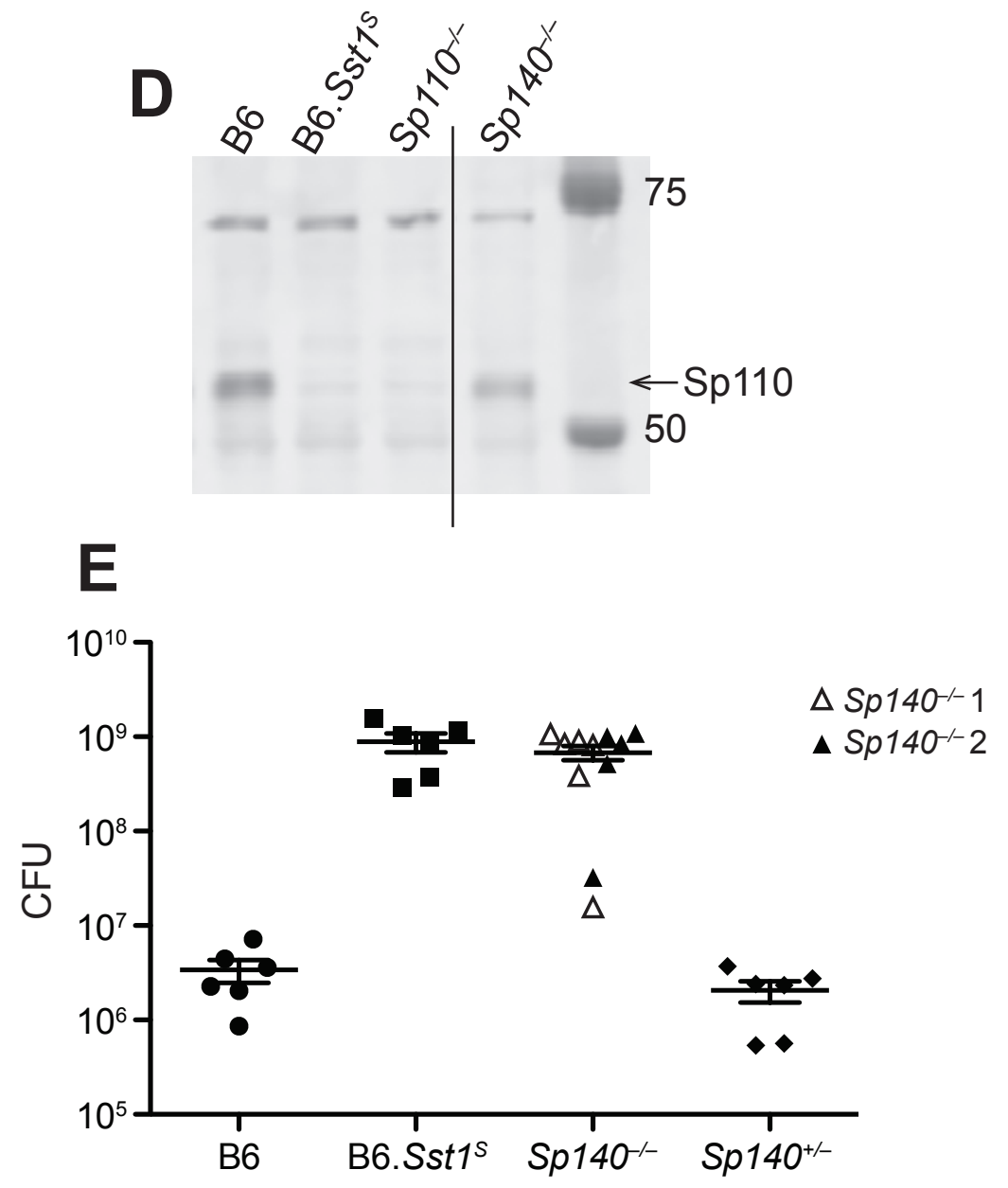

Figure S2. CRISPR/Cas9 targeting strategy for Sp140-- and validation of founders. (A) Mouse Sp140 gene. Guide RNA sequence for CRISPR/Cas9 targeting and protospacer-adjacent motif (PAM) are indicated. (B-C) Sp140 locus in wildtype (WT) and 2 independent founders of Sp140-- validated by sequencing. (D) Immunoblot for SP110 using BMMs from mice of the indicated genotypes. Intervening lanes have been removed for clarity (indicated by line in the image). (E) M. tuberculosis-infected mice were harvested for CFU at 25 days post-infection. Empty and filled triangles indicate the two independent lines of $S p 140^{--}$used in this infection. All mice were bred in-house and Sp140 ${ }^{+/-}$were littermates with Sp $140^{-1-} 2$. 